\title{
Probing the kinome
}

\section{Researchers are eager to map out all the signaling pathways regulated by protein kinases. Many new protocols and reagents are available to tackle this formidable task. Laura Bonetta reports.}

Phosphorylation is the main device a cell uses to control the function of its proteins. The responsible enzymes, protein kinases, regulate a myriad of processes from cell division in development to neuronal communication in memory. In addition, many of them have been implicated in disease. The notorious Novartis drug Gleevec, which has had great success in treating chronic myeloid leukemia and gastrointestinal stromal tumors, is a kinase inhibitor. It is no surprise then that academic labs and pharmaceutical companies are hot on the trail of understanding kinase function.

Kinases impart their signal by transferring the $\gamma$-phosphate group from ATP to a recipient serine, threonine or tyrosine residue on a protein. The choice of target is decided by the catalytic domain of the kinase, which usually recognizes a docking domain on the substrate and a specific, short sequence of amino acids (the consensus motif) that surround the residue to be phosphorylated. Each kinase can have several substrates and be itself a substrate for other kinases.

To start to delineate the complex network of pathways involving kinases, researchers want to match every kinase in a cell, representing the so-called kinome, to specific phosphorylation sites on target proteins. Products and assays to accomplish this ambitious task are making their way into research labs.

\section{Monitoring kinase activity}

Not every kinase will work in an in vitro assay and peptide that is phosphorylated by a kinase in vitro does not necessarily reflect that kinase's physiological target. However, kinase kits provide an efficient way to monitor the activity of a kinase and screen compounds that will affect this function. Many companies offer reagents for these tests, including off-the-shelf and custom-made kinase kits, phosphospecific antibodies, purified kinases, and specific substrates and inhibitors.

The initial kinase assays used radioactive ATP in the reaction mixture. But the trend in recent years has been to move away from using radioactivity. A researcher can now choose from a wide variety of fluorescence- and luminescence-based assays. Molecular Devices, for example, has a fluorescence polarization-based kinase assay that uses its proprietary IMAP technology. When fluorescently labeled peptides are incubated with a kinase, the phosphorylated peptides bind to the IMAP reagent-a trivalent metal with high affinity for phosphates-causing an increase in the polarization of the fluorescence. "The assay is highly resistant to interference and gives a strong signal," says Richard Sportsman, vice president of reagents development. For a new kinase of unknown specificity, Molecular Devices provides the IMAP Substrate Finder products, consisting of arrays of 55 different peptides in microwell-plate formats, which can be tested against the kinase. "The substrates are picked from all relevant regions of the kinome," says Sportsman. "Typically customers will get 3-4 hits. They can then order the best substrates for further testing."

Another platform offered by a number of companies is based on fluorescence resonance energy transfer (FRET) a detection method that relies on the proximity of two fluorescent dye molecules (fluorophores) to allow the transfer of excitation from donor to acceptor moiety. Invitrogen's Z-LYTE kinase assay uses synthetic peptides labeled with both donor and acceptor fluorophores. When a site-specific protease is added to the phosphorylation reaction, it cleaves non-

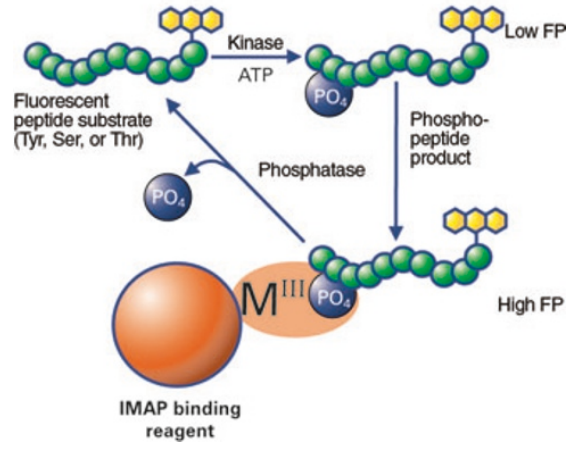

The IMAP technology from Molecular Devices is a homogeneous fluorescent kinase (or phosphatase) assay. Phosphorylation of a fluorescent substrate is detected by its binding to trivalent metals on nanoparticles and measuring the resulting increase in fluorescence polarization. (Courtesy of Molecular Devices.)

phosphorylated peptides causing them to lose the FRET signal. "We have developed a portfolio of substrates that have been validated with over 130 kinases and have the potential to be used to assay $85 \%$ of all kinases in Z-LYTE format," says Chris Armstrong, business area manager for drug discovery solutions.

Invitrogen also markets another assay based on time resolved (TR)-FRET called LanthaScreen, which can be used to study not only phosphorylation by kinases but a whole variety of protein interactions. An antibody labeled with a donor terbium fluorophore associates specifically with phosphorylated peptide substrates labeled with the acceptor fluorophore, resulting in an increased TR-FRET value in the reaction mixture. "We have tried to focus on generic reagents, so that a researcher can build his or her own assays." says Armstrong.

Promega markets a luminescencebased system, Kinase-Glo, which measures changes in ATP levels in a kinase 


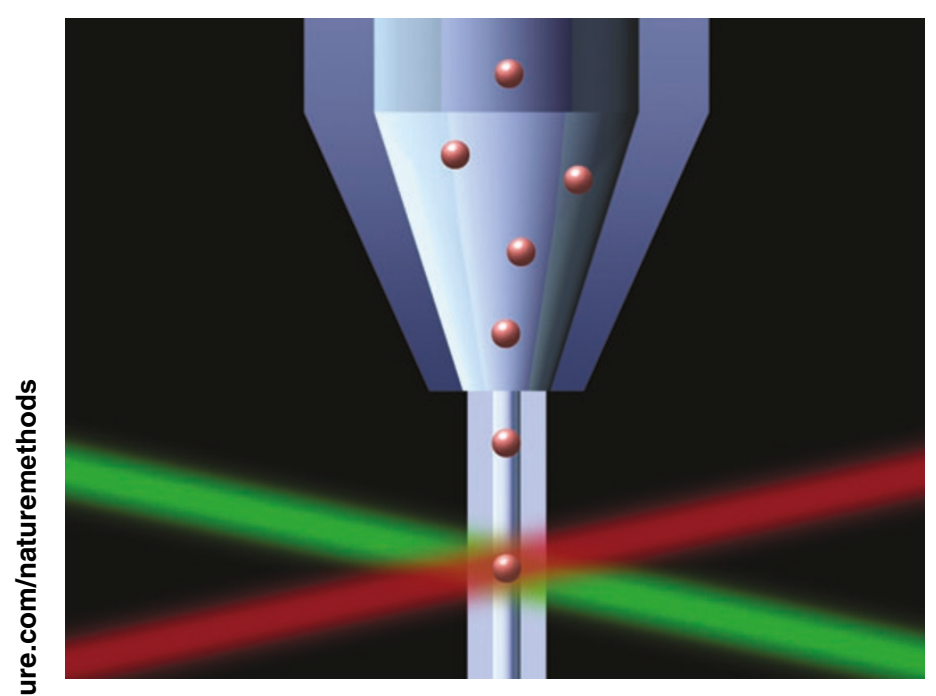

Dual laser detection using the Beadlyte technology. As each bead flows through a cuvette in a single file, a red laser detects the identity of the bead and a green laser quantifies the fluorescence associated with the analyte-bound reporter. (Courtesy of Upstate Group LLC.)

reaction. ATP from a completed kinase assay drives a reaction with luciferin and luciferase to produce oxyluciferin and light; the amount of light produced is inversely correlated with kinase activity. "The nice thing about this assay is that it works with a broad range of purified kinase-substrate combinations" says Michael Curtin, product manager for cellular analysis. "The substrate can be a peptide, protein, lipid, sugar-just about anything a kinase normally uses as a substrate".

\section{Identifying in vivo substrates}

The holy grail of kinome analysis is to determine the physiological substrates of all kinases to begin to map out signaling pathways. The KESTREL (kinase substrate tracking and elucidation) method offered by Kinasource identifies specific sequences that are phosphorylated in vivo. Cell lysates from many different tissues are incubated with a purified active kinase and the resulting phosphoproteins are separated by gel electrophoresis. Any leads identified in this initial screen are then pursued in a scaled-up protocol to isolate selected proteins from the gel and determine their identity by mass spectrometry. "Most researchers ask us to purify all the strong signals from the initial screen," says Axel Knebel, managing director of Kinasource. The initial screen typically requires $2-3$ weeks and the purification an additional month on average.

"In theory the experiment is quite easy to do, but, like mass spectrometry, you need to accumulate the experience and skill to 
do it," says Knebel. Kinasource provides KESTREL as a service to pharmaceutical companies and as a mix of collaboration and service to academic labs. "Academic researchers get a much better price than the pharmaceutical companies, but we end up on the published paper," says Knebel.

According to Knebel the method is successful in finding physiologically relevant substrates $80 \%$ of the time. "Some kinases don't bind ATP that well and cannot be used in the assay," he says. Another limitation is that KESTREL does not work well for scarce or rare substrates because the technique suffers from a relatively high level of background contamination.

In an effort to get around some of these problems other researchers are finessing methods that do not require gel electrophoresis. One such technique uses immobilized metal affinity chromatography (IMAC) resins with a high specificity for phosphates to purify phosphopeptides in bulk from protease-digested cell extracts. The isolated peptides are then identified by reversed-phase liquid chromatography and tandem mass spectrometry (LC-MS/MS).
To enrich for proteins that are phosphorylated on tyrosine-the minority of all phosphorylated proteins in a cellresearchers in Arthur Salomon's group at Brown University added an immunoprecipitation step using an antibody that specifically recognizes proteins phosphorylated on a tyrosine residue (Box 1) before purification by IMAC". "The goal is not to find the most phosphorylation sites. It is to find those associated with signaling pathways and disease in the shortest amount of time," says Salomon. "One-third of all proteins in a cell are phosphorylated, but most are not relevant to signaling. We tried to design a method to efficiently extract the most meaningful data by focusing on proteins phosphorylated on tyrosine."

As an alternative, the PhotoScan technology developed by John Rush et al. ${ }^{2}$ at Cell Signaling Technology (CST) uses a phosphotyrosine antibody immobilized on agarose beads to isolate phosphotyrosine-containing peptides from proteasedigested cell extracts, which were then analyzed by LC-MS/MS. The strategy offers "a simple procedure to selectively enrich for the tyrosine phosphoproteome," says Christopher Bunker, director of new business development at CST. "It avoids the limitations of IMAC methods such as charge bias, enrichment based on abundance and multistep procedures." According to Bunker, the company has identified nearly 4,000 phosphotyrosine sites, of which almost $70 \%$ are new.

To make these procedures amenable to large-scale screens, Salomon has automated many of the steps involved in processing samples and gathering data. "In one hour we can get 300 phosphopeptide mass spectra," says Salomon. The bottleneck, however, is having to interpret the information. A number of algorithms are available to match mass spectra with protein sequences but they "are not optimized for phosphopeptide spectra," says Salomon. "Manual validation of the spectra is still necessary." Another challenge is determining which phosphopeptides represent interesting substrates to pursue in follow-up experiments. "We have to do experiments like time courses to see which phosphorylation

\section{BOX 1 PHOSPHOSPECIFIC ANTIBODIES}

Phosphospecic antibodies recognize the phosphorylated form of specific amino acid sequences or consensus binding motifs, including some that are generic for either tyrosine or serine and threonine phosphorylation. Cell Signaling Technology was the first company to release these products on the market and currently stocks $500-600$ phosphospecific antibodies. "We make all of them in house, confirm the antibodies' performance in multiple applications and provide the exact protocol for using them - and we guarantee they will work," says Christopher Bunker, director for new business development.

Phosphosolutions, with an arsenal of about 60 phosphospecific antibodies, is focusing on the nervous system. "The phosphoantibody field has caught fire in cancer research but there are not that many phosphospecific antibodies for proteins in the nervous system," says company president Michael Browning. "Almost all kinases are enriched in the brain. It is a target-rich environment and at the moment we are just scratching the surface."

The antibodies are already being exploited for many different applications, including immunohistochemistry and flow cytometry, to monitor kinase activity in cells. "These antibodies will tell you what a protein is doing and where it is doing it," says Browning. In 3-5 years researchers hope to have phosphospecific antibodies on a chip to be able to simultaneously measure the phosphorylation state of dozens of distinct targets in a single sample. "The technology is there, but what we are missing is the content," says Browning. " 90 percent of the battle is having good, specific antibodies and lots of them." 
sites are changing and might point to interesting pathways," says Salomon.

\section{Screening libraries}

An alternative to finding sites that are phosphorylated in vivo is to use peptide libraries to determine the amino acid sequence preference of a given kinase and then draw on bioinformatics tools and databases to uncover candidate substrates that contain this sequence motif. In these protocols the kinase of interest is incubated in solution with many pools of peptides, all containing a single fixed serine, threonine or tyrosine residue within a sequence consisting of all possible amino acids. Only those peptides that contain an amino acid sequence favored by the kinase are phosphorylated. The phosphorylated peptides are then separated from the nonphosphorylated ones and sequenced. "You are asking the contribution of a given residue in a sequence motif based on how much it is enriched at each position in the peptide mixture," says Zhou (Sunny) Songyang at Baylor College of Medicine. A consensus motif is then generated in a statistical manner, such that the contribution

\section{BOX 2 SCANSITE}

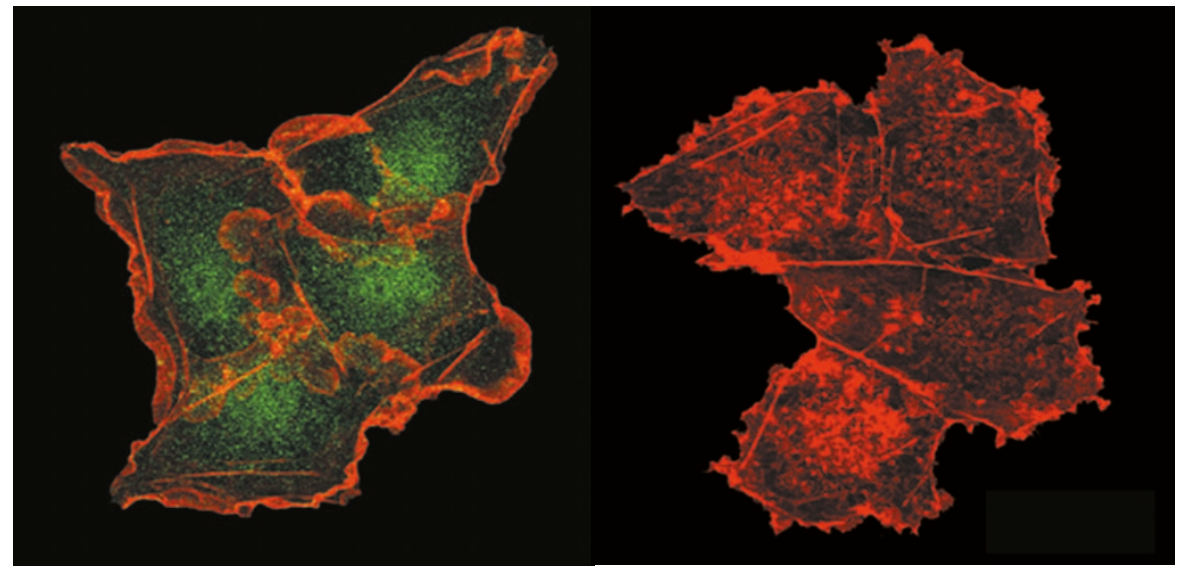

Immunofluorescent analysis of cells using an antibody specific for phosphorylation by the J NK kinase (green) and Texas Red X-conjugated phalloidin (red) to detect actin. The cells in the right panel are untreated. The cells in the left panel were treated with epidermal growth factor, which caused activation and nuclear localization of J NK and rearrangement of the actin cytoskeleton. (Courtesy of Cell Signaling Technology and Harry Mellor, University of Bristol, UK.) of each amino acid is weighed against that of all the others in the sequence (Box 2). The motif can then be exploited to scan protein databases in search of candidate substrates that can be further tested.

Recent refinements to the technique have made it better suited to
Say you have found a phosphorylated protein by mass spectrometry and you want to know where the phosphorylation sites are and which kinase might be responsible for phosphorylating them. Or maybe you have found a potential kinase consensus motif by screening a peptide library and want to know which proteins contain the motif. Scansite (http:/ / scansite.mit.edu) is a web-based tool designed to help with these kinds of analyses. "Our goal was never to make a public tool," says Michael Yaffe who codirects the site with Lewis Cantley. "We had started developing Scansite to make something to use for our own lab."

The current release of the site contains 63 motifs derived from oriented peptide library screens, each combined with a scoring matrix that indicates the preference for each amino acid at each position within the motif. A researcher can enter a protein sequence or ID and Scansite will identify all the motifs within the protein ranked in order of preference, indicating possible substrate-kinase interactions. To accommodate large proteomics experiments, "users can input up to 10,000 protein sequences at once," says J ohn C. Obenauer at St. J ude Children's Research Hospital. Scansite can also be used to scan through different protein databases to find proteins that contain a particular consensus motif. "It weighs and searches through computation rather than by text searching," says Songyang.

"You al ways find lots of hits but the sites may not be accessible depending on the conformation, for example, or the candidate substrate and kinase may be differentially localized in the cell," says Turk. "The challenge is to find matches between what has been done experimentally and the list obtained with Scansite." To help with this process, Scansite also links to Cell Signaling Technology's Phosphosite database (http:// www. phosphosite.org), a catalogue of in vivo phosphorylation sites. "If a protein is actually known to be phosphorylated in cells at a site identified in Scansite, it is a good candidate substrate," says Turk. high-throughput screens. In the oriented array peptide library (OPAL) approach, hundreds of pools of oriented peptide libraries are synthesized and arranged as scan arrays, in which each spot corresponds to a fixed amino acid at a particular position flanking the tyrosine, serine or threonine ${ }^{3}$. The pools of peptides are phosphorylated in situ and the amount of radioactivity at each spot on the membrane is determined. "OPAL cut the step of sequencing peptides so that you can do the procedure much faster," says Songyang.

Applying the same principle, Ben Turk at Yale University School of Medicine in collaboration with Lewis Cantley's group at Harvard Medical School synthesized 198 separate degenerate peptide libraries, each containing a fixed serine or threonine residue and another fixed amino acid in one of nine flanking positions ${ }^{4}$. However, unlike with the OPAL approach, the phosphorylation reactions were carried out in solution and libraries were later captured onto membranes. "We successfully profiled 40 kinases but the method did not work with 4 . Perhaps it will not work for $5-10 \%$ of all kinases," says Turk. He is planning to work with anaspec to resynthesize the library and make portions of it commercially available. "The library cost us about $\$ 30,000$ to make. We are trying to commercialize it so that it will be cheaper," he says.

The identification of a phosphorylation motif does not, however, directly lead to the identification of a protein substrate. "A 
kinase has to have a consensus sequence to phosphorylate a protein but the consensus does not in itself tell a protein 'I am a substrate.' You need to have the right conformation and other conditions," says Axel Knebel of Kinasource. "Many consensus motifs are known, but it is impossible to know in silico which will be substrates."

The key is to follow up the leads provided by these in vitro screens with the right experiments. "Once you have a list of candidate proteins you need to express them to show that the motif is phosphorylated in vivo," says Michael Yaffe at the Massachusetts Institute for Technology. "Two big advances in the field would be to have access to full length cDNAs that are tagged so that they can be expressed in cells and to protein arrays with 32,000 human proteins."

The consensus motif can also be used to generate antibodies to facilitate the search for substrates in vivo. Unlike phosphoantibodies raised against a single amino acid sequence, phosphomotif antibodies (first developed by Cell Signaling Technology) recognize many different phosphoproteins, as long as they are phosphorylated within a limited sequence context (Box 1). "We hope that in a matter of a few years we will have identified all the kinase motifs and generated phosphospecific antibodies to them," says Turk.

\section{Laying down the chips}

Much like the ever-popular DNA microarrays, peptide arrays provide a quick and easy test for profiling kinase function, requiring small quantities of reagents. Pepscan Technologies' PepChip Kinase contains 1,200 peptide substrates in duplicate on a single chip in standard microarray format. JPT Peptide Technologies synthesized arrays containing up to 11,000 phosphorylation-site peptides in triplicate that they screen as a service to pharmaceutical company clients-a smaller 720-peptide version of the array is sold commercially to individual labs. JPT has also adapted the microarray technology to search for phosphorylation sites within kinase substrate proteins.
The Phosphosite Detector array consists of overlapping peptides from a substrate. "You look for signals and then based on the alignment of the sequences determine which amino acid is phosphorylated," says JPT's Mike Schutkowski.

Researchers in the kinase field are eagerly awaiting the development of affordable protein arrays that represent the entire component of proteins in a human cell (proteome). Such arrays will provide an invaluable tool for testing a kinase or mixture of kinases against all possible protein targets. At the moment, several companies sell protein arrays that cover a fraction of the human proteome.

\section{Finding kinases for substrates}

Peptide screening methods start with a kinase and end up, if all goes well, with a candidate substrate to study. A recent publication by Kevan Shokat's group used the reverse approach ${ }^{5}$. Because many kinase substrate interactions are short lived, Shokat designed a new crosslinker that connects a substrate of interest to 
its upstream kinase, thereby facilitating the purification of the entire complex with antibodies.

Whereas the preliminary results are promising, many challenges remain. "We don't know the limitations of the procedure yet. We need to look at it more systematically," says Shokat. The crosslinker may soon find its way to the market place as several companies have expressed an interest in developing the product.

One of the exciting applications of the crosslinker method is that it can be used to validate results obtained with bioinformatics tools. "If you screen for candidate substrates of a kinase, the challenge is to confirm the hits. One way to do it is to crosslink each candidate protein and then look for the corresponding kinase in cell extracts," says Shokat. "What you are doing is mapping a pathway in one direction and then going back in the other."

Knocking out (or down) kinase function One of the more traditional methods for probing the function of a protein is to knock out its function and look at the effects in cells or whole animals. Methods to knock out kinase function include engineering genetic knockout animals and using siRNA or chemical inhibitors. Many companies provide the necessary reagents for these studies.

Genomatix Corporation has developed a different strategy. Their approach combines a subcellular localization signal with a mutant substrate 'decoy' that inhibits a kinase by competing with the wild-type substrate. The end result is the knockdown of kinase function in only one subcellular domain. "Our tool allows researchers to microdissect out the roles of a kinase in different cellular localizations," says chief science officer Thomas Reed. The company's mDECOY transgenes can be quickly assembled from a selection of genetic modules to the customer's specifications. "The vectors can then be used to study kinase activity in cells or to make transgenic animals," says Reed.

The system may have some advantages over methods that knock out (or down) kinase function throughout the cell. "Kinases are not only involved in signal transduction but also in regulating diverse effector proteins throughout the cell," says Reed. "By localizing the reagent to a specific cellular compartment scientists can begin to differentiate between these different roles.”

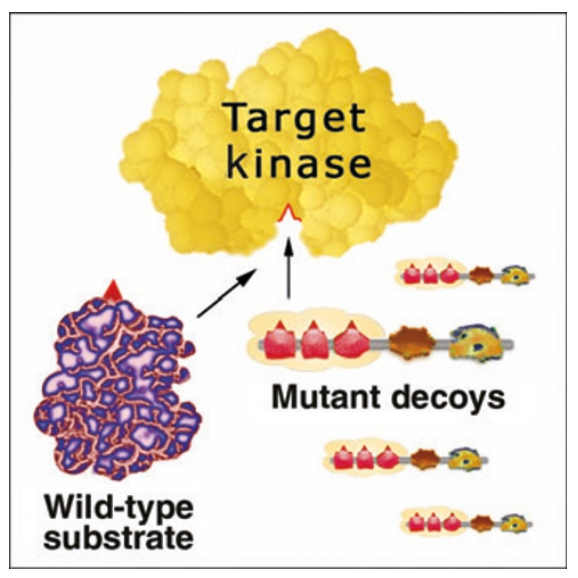

The competitive inhibition of the mDECOY approach. (Courtesy of Genomatix Corporation.)

Cellular Genetics Inc. (CGI) has developed a stock of genetically engineered kinases that are fully functional but can be inhibited in a specific, inducible manner by small molecule inhibitors. "The approach can be used to systematically generate conditional alleles of protein kinases allowing for rapid, functional characterization of kinase activity," says Shokat who pioneered the technology. CGI scientists use their reagents, including genetically modified mice carrying the mutant kinases, to provide a screening service to pharmaceutical companies to test inhibitors and find new substrates. "You look at all the phosphoproteins in a cell, then knock out a particular kinase and do a subtraction experiment. Any proteins that are no longer phosphorylated can be identified by mass spectrometry," says Shokat.

\section{Phosphorylation goes live}

Whereas kits for monitoring kinase activity in vitro are widely available, there are fewer choices for researchers looking for cell-based assays. Invitrogen has developed a panel of CellSensor live cell assays using its GeneBLAzer technology, which combines a $\beta$-lactamase reporter gene (bla) with FRET-based detection. A substrate labeled with two fluorophores is loaded into cells. In the absence of bla expression, the excitation of one fluorophore results in FRET to the other, causing it to emit green light. But, if a particular pathway is activated, thereby causing bla expression, the substrate is cleaved separating the two fluorophores. The result is 
a blue fluorescent cell. "You can challenge these cells with a kinase inhibitor and look at the effects on a particular biological pathway," says Armstrong. "Right now we have a focused collection of CellSensor lines which cover several key pathways in various cell backgrounds, but we plan to significantly increase the breadth and depth of our portfolio."

Another cell-based assay, Upstate's Beadlyte technology, works on the Luminex xMAP platform-an assay system based on fluorescently labeled beads and reporter molecules that can detect hundreds of soluble agents in the same assay well. Each Beadlyte bead is labeled with a protein or phosphospecific antibody that can specifically recognize kinases and their substrates in a cell extract. Another antibody labeled with a fluorescent reporter then binds the captured molecule. As the bead passes through the Luminex reader, one laser excites the bead to identify it, and another laser excites the fluorescent tag on the second antibody to quantitate the amount of captured analyte. "We can monitor multiple signal transduction pathways at once with our repertoire of state-specific monoclonal antibodies," says Till. "For example, we can apply this technique to follow the effects of kinase inhibitors in a physiologically relevant environment to identify off-target interactions." Right now Upstate provides this technology as a service to pharmaceutical clients, but is developing a 'desktop' version for researchers.

The family of human kinases is thought to have more than 500 members, of which only a fraction have been described thus far. But the future looks bright. As more cell-based assays become available and the number of phosphospecific antibodies and other reagents continues to grow, researchers will have access to a stockpile of powerful tools to decode the signal transduction pathways regulated by kinases.

1. Brill, L.M. et al. Anal. Chem. 76, 2763-2772 (2004).

2. Rush J. et al. Nat. Biotechnol. 23, 94-101 (2005).

3. Rodriguez, M. et al. J. Biol. Chem, 279, 8802 8807 (2004).

4. Hutti, J.E. et al. Nat. Methods 1, 27-29 (2004).

5. Maly, D.J. et al. J. Am. Chem. Soc. 126, 91609161 (2004).

Laura Bonetta is a freelance writer based in the Washington, DC area.

lbonetta@nasw.org 


\section{TECHNOLOGY FEATURE}

\section{SUPPLIERS GUIDEF COMPANIES OFFERING KINOME ANALYSIS PRODUCTS}

\section{Company}

Amersham Biosciences

AnaSpec

Applied Biosystems

BD Biosciences (Pharmingen)

Biaffin $\mathrm{GmbH}$

Biocan

Biorad

BioSource International

Biotrend Chemikalien $\mathrm{GmbH}$

BioVision AG

Calbiochem (EMD Biosciences)

Cambrex Corp.

CHEMICON

Cell Signaling Technology

Cellular Genomics Inc.

Clontech (EMD Biosciences)

DiscoveRx

EMD Biosciences

Exalpha Biologicals Inc

GE Healthcare

Genomatix Corp.

Globozymes

Imgenex

Invitrogen

J erini AG

Kinasource

MBL International Corporation

Molecular Devices

Molecular Probes (Invitrogen Detection Technologies)

New England Biolabs

Pepscan Systems

PerkinElmer

Pierce Biotechnology Inc.

Phosphosolutions

Promega Corp.

Rockland I mmunochemicals for Research

Stratagene

Stressgen Bioreagents

Upstate

USBiological

Qiagen

\section{Web Address}

http:// www4.amershambiosciences.com/

http:// www. anaspec.com/

http:// www. appliedbiosystems. com/

http:// www.bdbiosciences.com/ pharmingen/

http:// www.biaffin.com/

http:// www. biocan.com/

http:// www.bio-rad.com/

http:// www. biosource.com/

http:// www. biotrend.com/

http:// www. biovision-discovery. de/

http:// www. emdbiosciences. com/ html/ CBC/ home. html

http:// www.cambrex.com/ default.asp

http:// www.chemicon.com/

http:// www.cellsignal.com/

http:// www.cellulargenomics.com/

http:// www. bdbiosciences.com/ clontech/

http:// www. discoverx.com/

http:// www.emdbiosciences.com/

http:// www.exalpha.com/

http:// www. gehealthcare.com/ usen/index.html

http:// www.genomatix.com/ kinasemdecoys.cfm

http:// www.globozymes.com/

http:// www.imgenex.com/

http:// www.invitrogen.com/

http:// www.jerini.de/

http:// www. kinasource.co.uk/

http:// www.mblintl.com/mbli/

http:// www. moleculardevices.com/

http:// www. probes.com/

http:// www.neb.com/

http:// www. pepscan.nl/

http:// www. perkinelmer.com/

http:// www. piercenet.com/

http:// www. phosphosolutions.com/

http:// www. promega.com/

http:// www.rockland-inc.com/

http:// www. stratagene.com

http:// antibody.stressgen.com/

http:// www. upstate.com/

http:// www.usbio.net/

http:// www1.qiagen.com/ 